'Becada de Medicina Interna, Universidad de Chile, Hospital San Juan de Dios, Santiago de Chile.

${ }^{2}$ Escuela de Salud Pública de la Universidad de Chile. Santiago, Chile.

${ }^{3}$ Departamento de Medicina, Universidad de Chile. Hospital San Juan de Dios, Santiago de Chile.

Conflictos de intereses: los autores declararon no tenerlos.

Recibido el 28 de marzo de 2014, aceptado el 12 de noviembre de 2014.

Correspondencia a: Dra. Paulina Núñez F paulinanunez@gmail.com

\section{¿Se sustentan las Guías GES en trabajos científicos financiados por los fondos de CONICYT?}

\author{
PAULINA NÚÑEZ F. ${ }^{1}$, ADRIÁN TORRES C. ${ }^{2}$, RODOLFO ARMAS M. ${ }^{3}$
}

\section{Contribution of Chilean research to the formulation of national clinical guidelines}

Background: In Chile, 80 diseases were included in a health care system called Health Care Guarantees (GES) and clinical guidelines were elaborated for their management. Aim: To assess the scientific background of guidelines and if they were based on research financed by the Chilean National Commission for Science and Technology. Material and Methods: The references of the 82 guidelines developed for 80 diseases were reviewed, registering their number, authors, country of origin and funding source. Results: The guidelines had a total of 6,604 references. Of these, only 185 were Chilean (2.8\%) and five (0.08\%) originated from research financed by the National Commission for Science and Technology. Conclusions: The contribution of research funded by national agencies to the formulation of clinical guidelines is minimal.

(Rev Med Chile 2014; 142: 1540-1546)

Key words: Chile; Health Planning Guidelines; Health policy.
L as políticas públicas legítimas corresponden a cursos de acción y flujos de información relacionados con objetivos públicos definidos en forma democrática. Para que puedan ser consideradas como tales, tienen que haber sido generadas en el marco de los procedimientos, instituciones y organizaciones gubernamentales, ya que son acciones de gobierno que buscan la forma de dar respuesta a las demandas de la sociedad. Ellas son desarrolladas por el Estado, con la participación de la comunidad y del sector privado. Incluyen orientaciones o contenidos, instrumentos o mecanismos, definiciones o modificaciones institucionales y previsión de resultados. En tal sentido deben ser consideradas como un proceso decisional, o sea, como un conjunto de decisiones que se llevan a cabo a lo largo de un tiempo determinado y con una secuencia racional ${ }^{1}$.

El adecuado manejo de aspectos técnicos y políticos permite desarrollar políticas públicas de excelencia, siendo necesaria una interacción que permita la solución técnica aceptable del problema con la posibilidad política de realizarla. El diseño implica un análisis de las diversas formas técnicas de resolver los problemas, identificando los fines y los medios para alcanzarlos y considerando soluciones teóricas o experiencias de otros países ${ }^{1}$.

A comienzo de la década del 2000, el Ministerio de Salud y la Comisión Nacional de Investigación Científica y Tecnológica (CONICYT) hicieron en Chile los primeros esfuerzos por estimular la generación de evidencias científicas para aplicar políticas públicas de calidad. Lo que tuvo público apoyo de la Academia Chilena de Medicina ${ }^{2,3,4}$. Después de un período de estudios y acuerdos, en mayo de 2004, el Consejo Nacional de Investigación en Salud del Ministerio de Salud y CONICYT crearon el Fondo Nacional de Investigación y Desarrollo en Salud (FONIS) "para incentivar investigaciones cuyos resultados contribuyan a mejorar o implementar las políticas públicas sanitarias" 5 .

Por otra parte, la Ley 19.966, del 25 de agosto 
de 2004, estableció un régimen de garantías en salud (GES), que alcanza a un cierto número de afecciones que son priorizadas considerando diversos factores como son la efectividad de las intervenciones y su relación costo efectividad, la carga de enfermedad, evaluaciones económicas, demanda potencial, capacidad de oferta del sistema de salud chileno, etc. Con estos estudios, más la experiencia, evidencia nacional y extranjera, se establece la priorización con que ingresan las afecciones que abarca el sistema. Es así como a la fecha, se han incluido 80 patologías que tienen garantía de acceso universal. Por cada patología que se va incluyendo en el programa GES, se reúnen grupos de expertos convocados por el Ministerio de Salud, que realizan una acuciosa revisión bibliográfica y elaboran la guía clínica correspondiente, plasmada en un documento de acceso público.

Es interesante destacar que en el área asistencial hay una concentración clara de los recursos en patologías priorizadas, lo que no ocurre en el campo de la investigación. Este fue un aspecto que preocupó al nivel del Ministerio de Salud, donde se desarrolló un método de priorización de materias a investigar, pero que nunca se aplicó ${ }^{6}$.

Este trabajo tiene como propósito evaluar la participación de las investigaciones nacionales en el respaldo bibliográfico de las guías GES, precisando el número total de publicaciones en cada una de ellas y la proporción de éstas que son nacionales y las fuentes que las financió, tales como Fondo Nacional de Desarrollo Científico y Tecnológico (FONDECYT), Fondo de Fomento al Desarrollo Científico y Tecnológico (FONDEF) y Fondo Nacional de Investigación y Desarrollo en Salud (FONIS).

\section{Materiales y Método}

Se analizaron las guías de las 80 patologías incluidas en el GES publicadas por el Ministerio de Salud en su página $w e b^{7}$. Todas estas, en formato PDF, se encuentran agrupadas según año de incorporación, en $1^{\circ}$ régimen GES (julio de 2005 a julio de 2006), $2^{\circ}$ régimen GES (julio de 2006 a julio de 2007), $3^{\circ}$ régimen GES (julio de 2007 a julio de 2008), guías clínicas GES 2010 y guía de Medicina Preventiva (esta última se incorporó, por términos prácticos, en el régimen anterior dado que es sólo una guía clínica) y las nuevas patologías
GES que se incorporaron en julio de 2013, que son las últimas que se incluyeron.

Cada guía fue evaluada por: 1) Número total de referencias bibliográficas; 2) Identificación de estudios chilenos publicados dentro del total de sus referencias; 3 ) Identificación de estudios con participación en fondos concursables (FONDECYT, FONDEF y FONIS) estipulado en la bibliografía. En cuanto a publicaciones nacionales, se registró: Lugar de publicación (revista nacional, extranjera) y área o especialidad de la Medicina; 4) Nacionalidad de los autores según lugar donde se realizó el estudio y la información que entregan los autores al publicar el documento. Esta búsqueda fue realizada en www.pubmed.gov (buscador de la NCBI US National Library of Medicine de los National Institutes of Health). Además, para evaluar publicaciones latinoamericanas y chilenas se utilizó SciELO (Scientific Electronic Library Online) o reconociendo las revistas nacionales.

Se excluyeron pautas, guías clínicas, capítulos de libros, revisiones sin lugar de publicación y normativas, dado que no disponían de la bibliografía utilizada. Además de capacitaciones, protocolos o participación en congresos que no identificaban un resumen o título de la presentación (Figura 1).

\section{Resultados}

En total, hay disponibles 82 guías de las 80 Patologías GES (más medicina preventiva y peritoneo diálisis), de las cuales se encuentra bibliografía en 81 de ellas. La única que no estaba disponible fue la guía clínica para el tratamiento de la hipoacusia en menores de 2 años, que se encuentra en la incorporación reciente de las nuevas patologías GES 2013. Por lo tanto, la disponibilidad de la bibliografía consultada fue $98,7 \%$.

Las referencias revisadas fueron en total 6.604 publicaciones, de las cuales, 185 fueron estudios chilenos de acuerdo a los criterios señalados. En este punto se tomaron en consideración los criterios de exclusión expuestos previamente, representando 2,8\% del total de estudios analizados.

En cuanto a publicaciones que tenían expresa participación de fondos (FONDECYT, FONDEF y FONIS), fueron citadas en las guías en 8 oportunidades, representando $0,12 \%$ del total de publicaciones. Cabe destacar que en las guías evaluadas, 3 de ellas nombran al proyecto de la Universidad de Talca de los factores de riesgo 


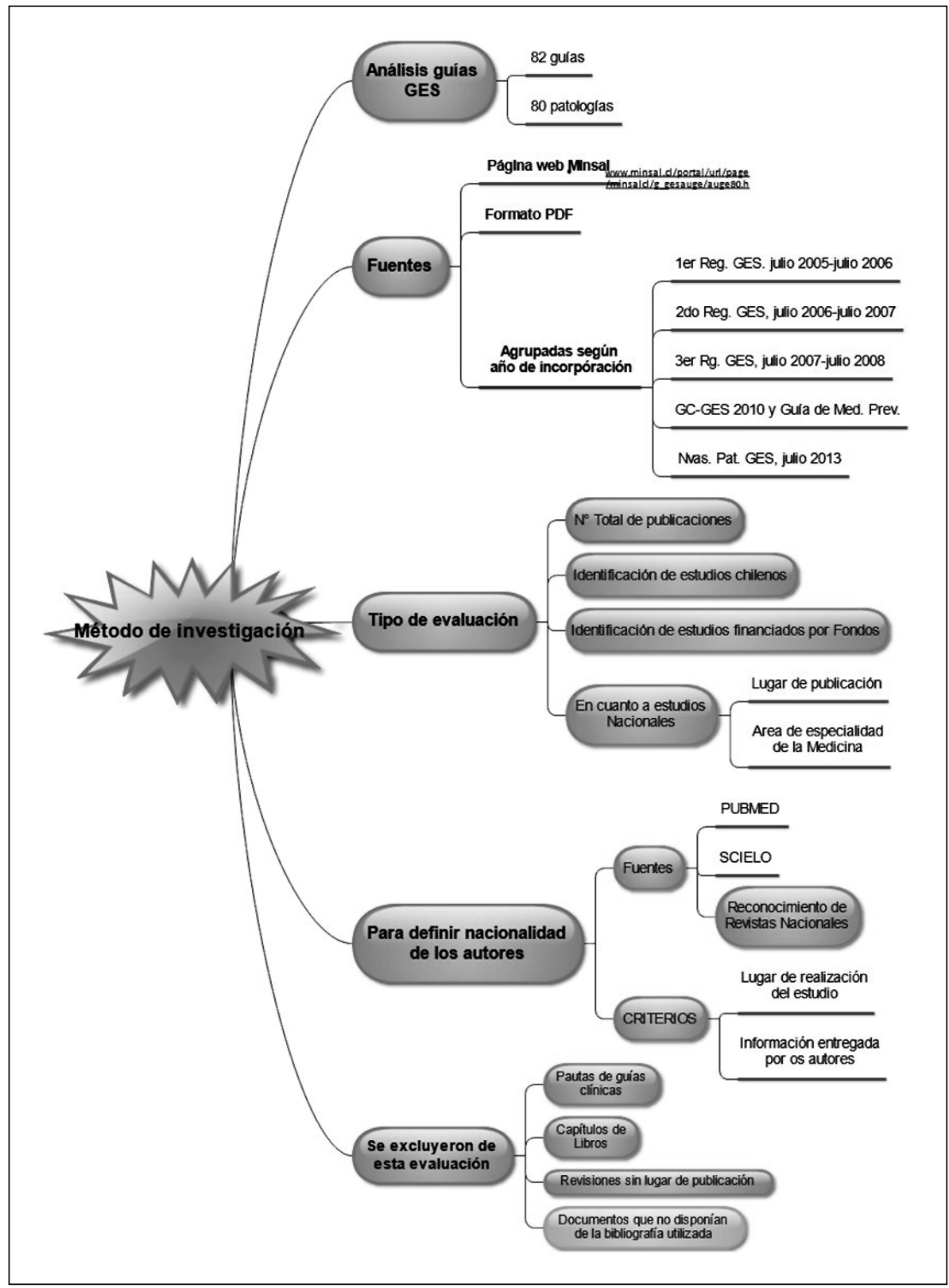

Figura 1. Resumen metodología empleada.

de enfermedades cardiovasculares dentro de su bibliografía, siendo en definitiva sólo 5 proyectos financiados los que participan en la elaboración de las guías, representando $0,08 \%$ de la bibliografía total analizada (Figura 2).

En 9 de las guías se señala que, aparte del grupo de expertos que se reunieron para la elaboración de estas, se solicitó un estudio externo para re- colectar bibliografía atingente a la realización de la guía, siendo 5 de éstas solicitadas en el último régimen GES. Los estudios externos, que están expresamente citados en la bibliografía analizada, fueron encargados a Medwave en las patologías: osteosarcoma en mayores de 15 años, cáncer colorectal en mayores de 15 años y más, cáncer de ovario epitelial; Sociedad Chilena de Cardiología 


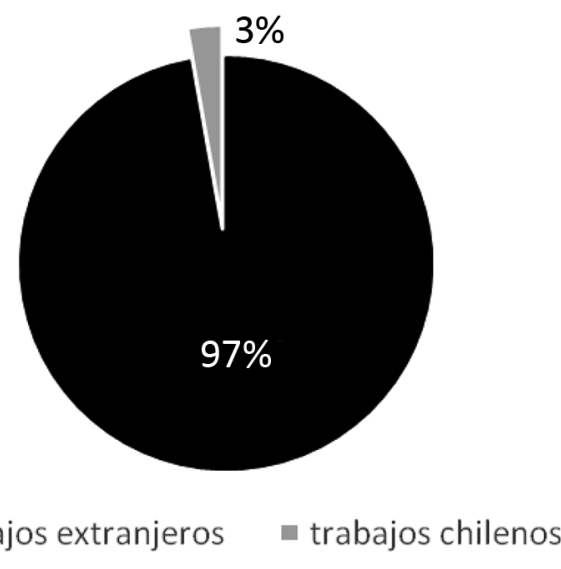

Figura 2. Distribución Publicaciones (Nacionales/Extranjeras/Fondos concursables).

(Sochicar) en patología valvular, Universidad del Desarrollo en hernia núcleo pulposo y asma bronquial, Universidad de Chile en el caso de cáncer gástrico y la Pontificia Universidad Católica de Chile, citada en la guía de neumonía adquirida en la comunidad.

Al tomar en consideración sólo los estudios nacionales, de las 82 guías disponibles, 44 de estas tenían algún estudio nacional publicado dentro de su bibliografía $(53,6 \%)$.

La guía clínica que tuvo el mayor número de referencias fue la de trastorno bipolar, con 285, seguida por la de cáncer de mama con 271, y tratamiento de personas con depresión con 266. De aquellas publicaciones en que se contó con la bibliografía, las que tienen un menor número de referencias, en forma decreciente, son: Guía de prematurez I: retinopatía del prematuro, Órtesis (o ayudas técnicas en mayores de 65 años) y
Epilepsia en el adulto con 11, 7 y 2 referencias, respectivamente.

En cuanto al número total de publicaciones nacionales, fueron más citadas en las guías de tratamiento de personas con depresión ${ }^{27}$, manejo y tratamiento de la infección por virus $\mathrm{B}$ de la hepatitis ${ }^{12}$, manejo de la infección por virus de la hepatitis C y Medicina Preventiva, ambas con 11 publicaciones nacionales.

Si realizamos un análisis por los tipos de régimen GES (Tabla 1), observamos que:

El mayor número de guías clínicas fue en el $1^{\circ}$ régimen, con 28 de éstas, y la de menor número la guía del año 2013 con 10. Llama la atención que dentro de las guías del $2^{\circ}$ régimen y del año 2013 no hay ninguna mención a publicaciones en base a fondos concursables y en el caso del último régimen es el que concentra el menor número de publicaciones nacionales.

Tabla 1. Revisión bibliografía disponible en guías clínicas por régimen GES

\begin{tabular}{|lccccc|}
\hline Guías GES & n guías & $\begin{array}{c}\text { n total de } \\
\text { estudios }\end{array}$ & $\begin{array}{c}\text { Estudios } \\
\text { chilenos }\end{array}$ & $\begin{array}{c}\text { Fondos } \\
\text { concursables }\end{array}$ & $\begin{array}{c}\text { Estudios } \\
\text { externos }\end{array}$ \\
\hline $1^{\text {er régimen }}$ & 28 & 2.082 & 51 & 4 & 1 \\
\hline $2^{\circ}$ régimen & 14 & 1.049 & 46 & 0 & 2 \\
\hline $3^{\text {er régimen }}$ & 18 & 1.160 & 12 & 2 & 1 \\
\hline Guías 2010 & 12 & 1.251 & 50 & 2 & 0 \\
Guías 2013 & 10 & 1.062 & 26 & 0 & 5 \\
\hline Total & 82 & 6.604 & 185 & 8 & 9 \\
\hline
\end{tabular}


Tabla 2. Estudios nacionales incluidos en guías clínicas según lugar de publicación

\begin{tabular}{|lccc|}
\hline Guías GES & $\begin{array}{c}\text { Revista } \\
\text { Médica de } \\
\text { Chile }\end{array}$ & $\begin{array}{c}\text { Otras } \\
\text { revistas } \\
\text { nacionales }\end{array}$ & $\begin{array}{c}\text { Revistas } \\
\text { extranjeras }\end{array}$ \\
\hline 1'r régimen & 10 & 32 & 9 \\
\hline 2 régimen & 19 & 16 & 11 \\
\hline 3er régimen & 1 & 8 & 3 \\
\hline Guías 2010 & 21 & 18 & 11 \\
\hline Guías 2013 & 16 & 3 & 7 \\
\hline Total & 67 & 77 & 41 \\
\hline
\end{tabular}

Tabla 3. Lugar de financiamiento de las publicaciones incluidas en guías clínicas

\begin{tabular}{|lccc|}
\hline Guías GES & Fondef & Fonis & Fondecyt \\
\hline $1^{\text {er régimen }}$ & 1 & 3 & 0 \\
$2^{\circ}$ régimen & 0 & 0 & 0 \\
\hline $3^{\text {er régimen }}$ & 1 & 0 & 1 \\
Guías 2010 & 0 & 2 & 0 \\
Guías 2013 & 0 & 0 & 0 \\
\hline Total & 2 & 5 & 1 \\
\hline
\end{tabular}

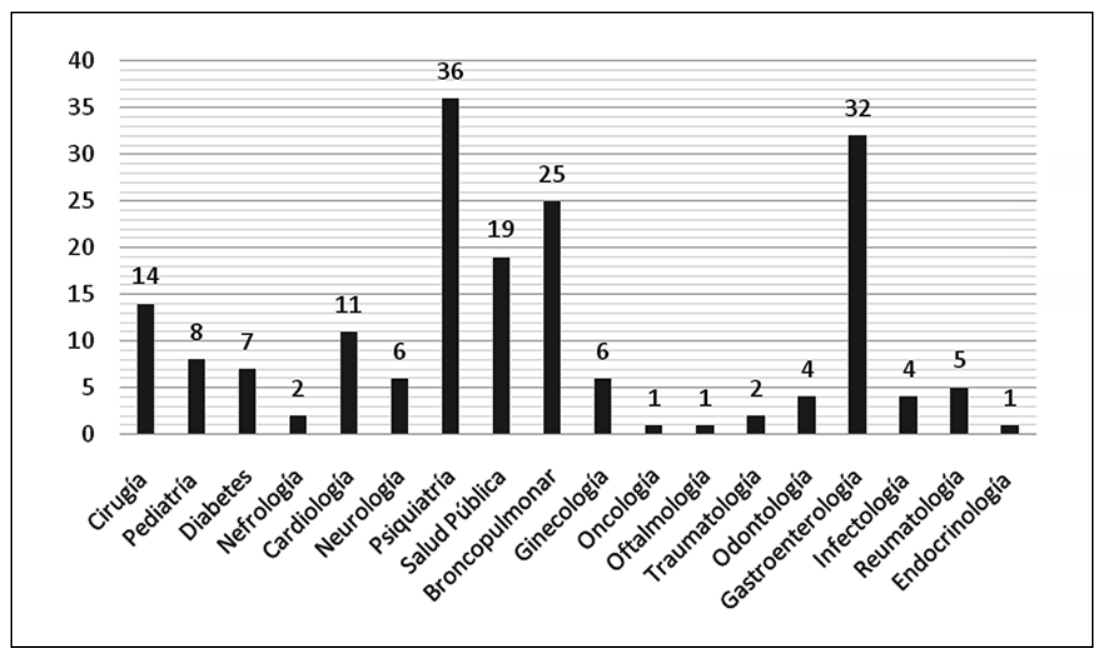

Figura 3. Especialidades médicas en publicaciones nacionales.

De las 185 publicaciones nacionales, 67 (31\%) eran de la Revista Médica de Chile, 41 (22\%) en revistas extranjeras y $77(43 \%)$ en otras revistas nacionales (Tabla 2).

En cuanto a las publicaciones financiadas por fondos concursables (dichos datos se muestran en la Tabla 3).

Cabe destacar que en 2 de las guías del primer régimen y una del año 2010 está dentro de sus referencias el proyecto de FONIS sobre Programa de Investigación de Factores de Riesgo de Enfermedades Cardiovasculares (PIFRECV), Universidad de Talca. Por lo tanto, en forma final corresponden a 2 FONDEF, 2 FONIS y 1 FONDECYT.

El análisis posterior fue ver de qué área o especialidad de la Medicina venían estas publicaciones nacionales, destacando Psiquiatría (36),
Gastroenterología (32), Broncopulmonar (25) y Salud Pública con (19) publicaciones (Figura 3).

\section{Discusión}

Los fondos de investigación nacionales no están generando evidencias para la formulación de las guías GES, particularmente llama la atención que los estudios financiados por FONIS -que se creó para fortalecer la investigación aplicada nacional en salud- no aporten ese respaldo científico. Es posible que en esto haya múltiples factores en juego y que en algunas especialidades actúen unos $y$ en otras otros.

Por cierto que es posible que haya especialidades en las que no existe actividad investigativa importante y, en tal caso, pareciera razonable buscar 
Sustento científico de las guías de garantías en salud, Chile - P. Núñez et al

algún tipo de incentivos para que se desarrollen, sea creando concursos con priorización temática, concursos específicos, formación de clínicos investigadores, implementación especial de centros, etc. Hay que tener presente que el trabajo clínico que se desarolla junto a un ambiente de investigación es habitualmente de mejor calidad que el que se desarrolla distante de ese ambiente. También es posible que en algunas disciplinas haya una dis- tancia laboral importante entre grupos que hacen investigación y grupos que sólo cultivan un trabajo clínico práctico y que no haya transferencia entre ellos; si ello ocurriese habría que buscar caminos para una mayor integración y mejor tranferencia entre esos grupos.

Probablemente la tendencia creciente dentro del trabajo clínico hospitalario con horarios pequeños atenta contra la investigación.

Anexo 1. Análisis de resultados I

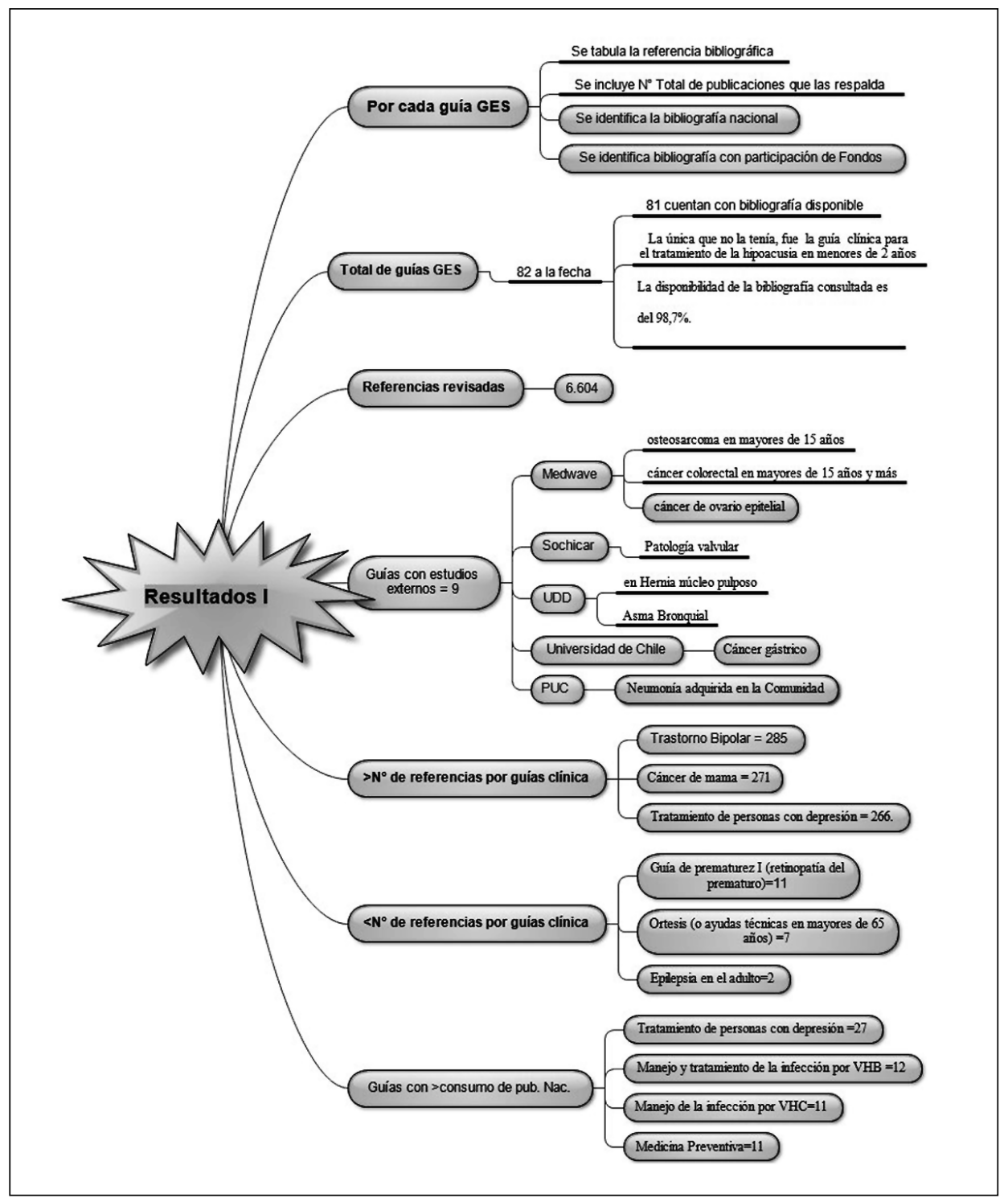


Anexo 2. Análisis de resultados II

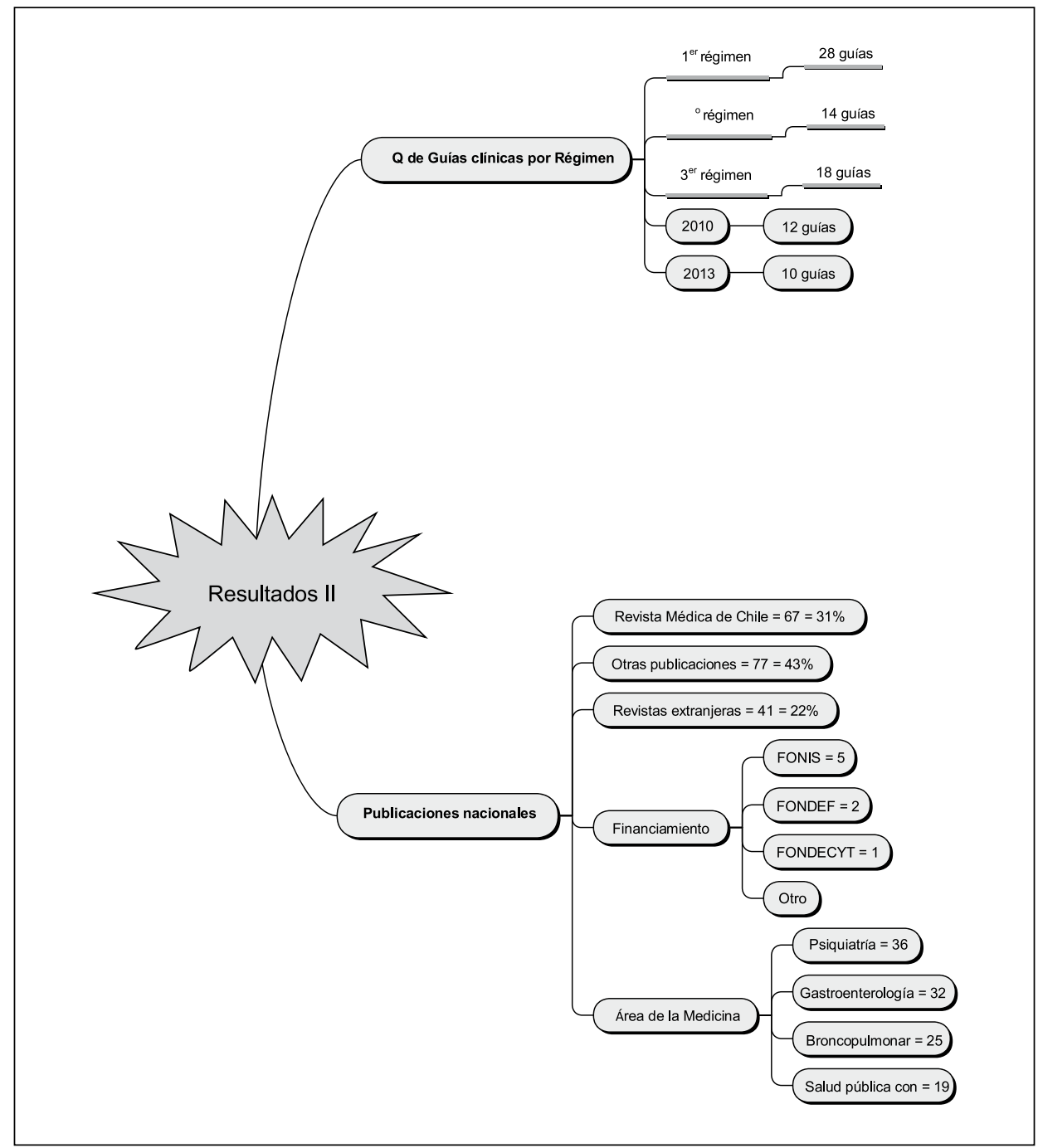

\section{Referencias}

1. Brieva HJ, Torres CA. Políticas Públicas para la Educación. Santiago de Chile: Ediciones Copygraph-IEDE 2010.

2. Armas MR. Hacia un Fondo Nacional de Investigación Aplicada en Salud. Boletín de la Academia Chilena de Medicina, Instituto de Chile 2001; 38: 95-998.

3. Valdivieso V. El futuro de la investigación en salud. Visión desde las Universidades. Boletín de la Academia Chilena de Medicina, Instituto de Chile 2001; 38: 89-93.

4. Academia de Medicina de Chile. Hacia un Fondo Sectorial de investigación en Salud. Documento de Posición.
Boletín de la Academia Chilena de Medicina, Instituto de Chile, 2001; 38: 229-30.

5. FONIS, ACTA $N^{\circ} 1$, Reunión Fundacional del Fondo Nacional de Investigación y Desarrollo en Salud. Santiago. 19 de julio de 2002.

6. Armas MR, Torres CA, Arriagada CJ, Muñoz PF, Salinas RR, Crocco AP. Proceso para priorizar las líneas de investigación esencial de interés para el Ministerio de Salud de Chile. Rev Med Chile 2001; 401-5.

7. Ministerio de Salud de Chile. http://www.minsal.cl. Obtenido de http://www.minsal.cl/portal/url/page/ minsalcl/g_gesauge/auge $80 . h t m l$ [Consultado el 8 de julio de 2013]. 